\title{
Prevalence Du Tabagisme Et Profil Des Eleves \\ Fumeurs Aux Lycees De Mahajanga I En 2014 (Madagascar)
}

\author{
Narindrarimanana Avisoa Randriamihangy \\ Adamo Ben Allaoui \\ Université de Mahajanga,
}

Service de Cardiologie du CHU Mahavoky Atsimo, Mahajanga, Madagascar

Freddie Raveloson

Université d'Antananarivo,

Service de Cardiologie du CHU JRB, Antananarivo, Madagascar

\section{Rondro Nirina Raharimanana}

Université de Mahajanga,

Service de Pneumologie du CHU P-ZAGA, Mahajanga, Madagascar

doi: 10.19044/esj.2016.v12n23p298 URL:http://dx.doi.org/10.19044/esj.2016.v12n23p298

\begin{abstract}
Introduction: Smoking is the most important cardiovascular risk factor among teenagers. Smoking often settles in adolescence and persists in adulthood. We aimed to determine the prevalence of smoking among high school students in Mahajanga I in 2014 and to describe their profile and attitudes toward smoking.
\end{abstract}

Methods: This is an analytical epidemiological cross-sectional study conducted in six high schools in Mahajanga I during November and December in 2014.

Results: Smoking prevalence was $6.6 \%$ and $36.82 \%$ of the students had smoked at least once in their lives. Furthermore, $64.22 \%$ started smoking by "tasting", $12.78 \%$ because of "influence" and 9.58\% used tobacco as a fashion. Most (74.71\%) of former smokers stopped smoking for fear of diseases; $40.86 \%$ due to the ban and $17.90 \%$ to lack of money. The half of active smokers was moderately to strongly addicted to nicotine. Tobacco price was rather reasonable, and even cheap for respectively $35 \%$ and $25 \%$ of students.

Some factors were significantly related to tobacco consumption: male gender $(\mathrm{p}=0.000)$, student's entourage as close friend $(\mathrm{p}=0.000)$, cousin $(\mathrm{p}=$ $0.000)$, brother $(p=0.000)$, uncle $(p=0.000)$, religious leader $(p=0.001)$, father $(p=0.002)$ and teacher $(p=0.005)$, being of age $(p=0.004)$ and ancestral religion $(\mathrm{p}=0.036)$. 
Conclusion: Our findings were quite encouraging with a lower smoking prevalence than in other studies elsewhere. The determining factors of smoking found in this study confirm the crucial role of both education and people around the student.

Keywords: Smoking, student, prevalence

\section{Résumé :}

Introduction : Le tabagisme est le facteur de risque cardiovasculaire le plus important chez le jeune. Notre objectif était de déterminer la prévalence du tabagisme auprès des lycéens et de décrire leur profil et comportement face au tabagisme.

Méthode : C’est une étude transversale épidémio-analytique, réalisée dans 6/26 lycées de Mahajanga I, par échantillonnage en grappe, en novembre et décembre 2014.

Résultats : La prévalence du tabagisme actif était de 6,6\% et 36,82\% des lycéens ont déjà gouté à la cigarette. Les lycéens avaient commencé à fumer pour « essai » chez 64,22\%, par « influence » chez 12,78\% ou pour la mode chez 9,58\%. Parmi les ex-fumeurs, 74,71\% avaient arrêté par peur des maladies, $40,86 \%$ par interdiction et $17,90 \%$ par manque d'argent. Une dépendance au tabac était observée chez $50 \%$ des fumeurs. Le prix des cigarettes était abordable pour 35\% des lycéens et bon marché pour 25\%.

Certains facteurs étaient associés à la consommation tabaquique : genre masculin ( $p=0,000)$, entourage fumeur tels que ami proche $(p=0,000)$, cousin $(p=0,000)$, frère $(p=0,000)$, oncle $(p=0,000)$, dirigeant religieux $(p$ $=0,001)$, père $(p=0,002)$ et enseignant $(p=0,005)$, âge $\geq 18$ ans $(p=$ $0,004)$ et religion traditionnelle $(p=0,036)$.

Conclusion : La prévalence du tabagisme chez nos lycéens était plus faible que celles de la plupart des autres villes et pays. Les facteurs déterminants du tabagisme confirment le rôle primordial de l'éducation et de l'entourage du lycéen.

Mots clés : Tabagisme, lycéen, prévalence

\section{Introduction}

Selon l'OMS, le tabac tue environ 6 millions de personnes chaque année, soit la moitié de ses consommateurs. En plus, 80\% des fumeurs vivent dans les pays comme le nôtre, à revenu bas ou moyen (WHO, 2014). Généralement, le tabagisme s’installe pendant l'adolescence et perdure chez les adultes. Le tabagisme constitue le facteur de risque cardiovasculaire le plus important chez les jeunes (Bejot, 2007) qui sont particulièrement fragiles devant les multiples stratégies de l'industrie du tabac et l'influence de leur entourage. 
Notre étude a pour objectifs de déterminer la prévalence du tabagisme chez les lycéens de Mahajanga I et de décrire leur profil et comportement face au tabagisme.

\section{Méthode}

Notre étude a été réalisée dans la ville de Mahajanga I, une ville cosmopolite située sur la côte Ouest de Madagascar. La population est jeune avec 50\% de moins de 20 ans et une prédominance féminine. Le nombre d'habitants était estimé à 187668 en 2013 (CREAM, 2014).

Il s’agissait d'une étude épidémio-analytique transversale, durant les mois de novembre et décembre 2014. Nous avons étudié la population des lycéens, au nombre de 6722 et répartis sur 26 lycées.Un échantillonnage en grappe a été réalisé avec un niveau de confiance à 95\%. La taille d'échantillon était calculée à 806,4 avec l'effet du plan d'échantillonnage et l'impondérable. Nous avons groupé les 26 lycées selon leur statut confessionnel. Les deux lycées publics ont été choisis d'office. Au total, 6 classes de seconde, 6 classes de première et 6 classes de terminale ont été tirés au sort. Les élèves ont reçu un questionnaire, à remplir en classe en présence de l'enquêteur. Celui-ci pouvait intervenir à tout moment pour apporter plus d'explication.

Nous avons inclus tout lycéen souhaitant remplir le questionnaire, après avoir reçu les explications. Les fiches mal remplies ont été exclues.

Les paramètres étudiés étaient l’âge, le genre, la religion, le statut du lycée, les attitudes des lycéens face au tabac, la situation matrimoniale des parents, le statut tabagique de l'entourage des lycéens, leur mode de vie (personnes vivant avec eux), leur connaissance sur les maladies liées au tabagisme, la dépendance tabagique, les dispositions prises au lycée face au tabagisme.

Etait considéré comme fumeur actif l'élève qui fumait régulièrement et répondait oui à la question "êtes-vous fumeur actuellement ? ». L’élève qui avait déjà fumé, même une seule fois dans sa vie, était étiqueté comme « ayant déjà fumé ».

Le score du test de Fagerström (INPES, 2015) était utilisé pour évaluer la dépendance à la nicotine : de 0 à 2 : fumeur non dépendant ; de 3 à 4 : fumeur faiblement dépendant; de 5 à 6 : fumeur moyennement dépendant ; 7 à 10 : fumeur fortement dépendant.

Une personne de l'entourage qui fumait ou qui chiquait du tabac régulièrement était considérée comme tabagique.

Pour le traitement des données, nous avons utilisé le logiciel IBM $^{\circledR}$ SPSS $^{\circledR}$ Statistics 20 et le logiciel Microsoft ${ }^{\circledR}$ Excel $^{\circledR}$ 2013. Nous avons utilisé le Test de Khi 2 de Pearson pour tester la corrélation avec comme seuil 
significatif une valeur de $\mathrm{p}<0,05$. Pour la force de corrélation, nous avons utilisé le test de $\mathrm{V}$ de Cramer. Une valeur supérieure à 70\% permettait de définir une forte corrélation.

\section{Résultats}

Au total 855 questionnaires ont été distribués et 850 correctement remplis avec un taux de participation de 99,42\%.

\section{Caracteristiques socio-demographiques de nos lyceens}

L’âge moyen était de 16,81 ans (12 à 24 ans). La sex-ratio était de 5/6 $(0,83)$ avec 386 garçons $(45,41 \%)$ et 464 filles $(56,59 \%)$.

Leurs religions étaient réparties comme suit : $77,76 \%$ de religion chrétienne, $19,65 \%$ de religion musulmane, $1,76 \%$ de religion traditionnelle malagasy et $0,82 \%$ ne se prononçaient pas.

Par rapport au statut du lycée, 47,88\% des élèves étaient issus de lycées publics, 9,65\% de lycées privés laïques, 16,24\% de lycées catholiques, 9,29\% de lycées musulmans et 16,94\% de lycées protestants.

\section{Prévalences}

a. Au moins une fois dans leur vie, $36,82 \%(n=313)$ des lycéens avaient expérimenté le tabac.

b. La prévalence des fumeurs actifs était $6,59 \%(n=56)$ avec une prédominance masculine, soit $10,88 \%$ des garçons contre $3,02 \%$ des filles ( $\mathbf{p}=\mathbf{0 , 0 0 0})$.

L’âge moyen de ces fumeurs actifs était de 17,48 ans (13 à 22 ans). La tranche d’âges de 15 à 20 ans représentait $80 \%$ des fumeurs actifs. Plus de la moitié des fumeurs actifs (51,8\%) étaient des mineurs. Mais 10,11\% des élèves majeurs contre $4,97 \%$ des élèves mineurs étaient fumeurs actifs ( $\mathrm{p}$ $=0,005)$.

L’âge moyen de la première prise de cigarette était de 13,15 ans (5 à 20 ans) et 14,05\% des élèves étaient initiés au tabac avant l’âge de 10 ans.

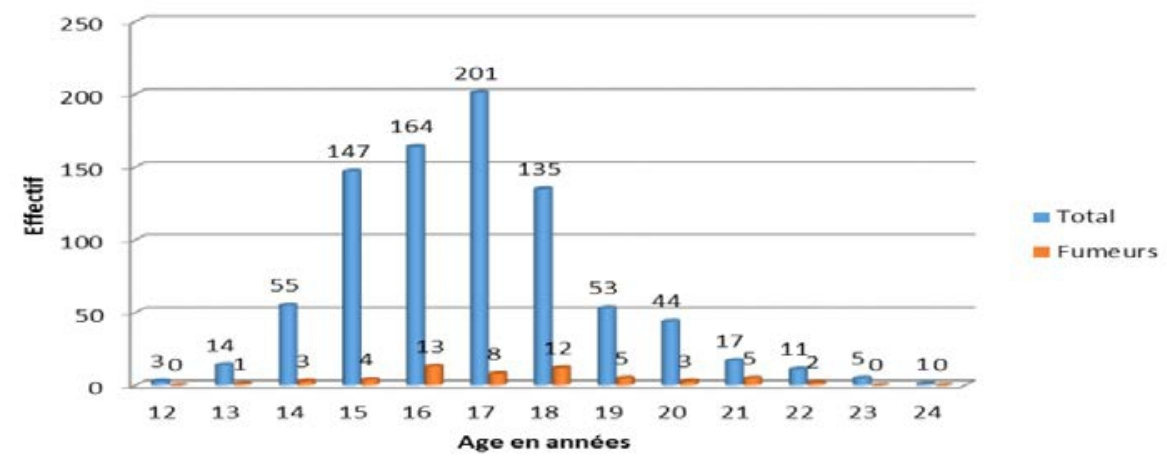

Figure 1 : Nombre des lycéens et des fumeurs actifs selon l'âge 
c. Pourquoi et ou les lycéens fumaient-ils ?

Les raisons ayant incité les lycéens à fumer étaient : l’essai (64,22\%), l'influence de l'entourage (12,78\%), la mode (9,58\%), la conviction (5,75\%), le fun $(5,75 \%)$ et l'interdiction (1,92\%).

Les lycéens fumaient dans différents endroits : 21,41\% ont cité la rue, 20,45\% chez eux, 12,46\% les espaces publiques et 4,79\% le lycée.

\section{Raison d'arret du tabagisme pour les ex-fumeurs}

Les lycéens qui avaient arrêté de fumer étaient au nombre de 257. Les raisons d'arrêt de leur consommation de tabac étaient les suivantes : la peur des maladies liées au tabac citée par 74,71\%, l’interdiction par 40,86\%, le manque d'argent par $17,90 \%$ et $1,95 \%$ ont répondu ne pas aimer fumer.

\section{Achat des cigarettes}

Les lycéens achetaient leurs cigarettes à 92,4\% dans les épiceries, à $7,6 \%$ au bord de la rue. Les fumeurs trouvaient le prix des cigarettes bon marché (25\%), abordable (35\%) ou cher (40\%).

\section{d. Dépendance au tabac}

Selon le test de Fagerström, 50\% de nos fumeurs actifs n’étaient pas dépendants au tabac, $25 \%$ étaient faiblement dépendant, 16,1\% moyennement dépendants et 8,9\% fortement dépendants.

\section{Facteurs determinants du tabagisme}

Nous avons classé les potentiels facteurs déterminants du tabagisme en deux : l'entourage fumeur et les autres facteurs (Tableau I et Tableau II).

Tableau I : Influence d'un entourage fumeur sur le lycéen

\begin{tabular}{ccccc}
\hline Entourage fumeur & $\mathrm{n}$ & $\begin{array}{c}\text { Lycéens fumeurs ou } \\
\text { ayant fumé } \\
313(36,82 \%)\end{array}$ & $\mathrm{p}$ & $\mathrm{V}$ de Cramer \% \\
\hline Ami proche & 285 & $148(51,93 \%)$ & $\mathbf{0 , 0 0 0}$ & $22,20 \%$ \\
Cousin & 315 & $148(46,98 \%)$ & $\mathbf{0 , 0 0 0}$ & $16,30 \%$ \\
Frère & 135 & $74(54,81 \%)$ & $\mathbf{0 , 0 0 0}$ & $16,20 \%$ \\
Oncle & 405 & $174(42,96 \%)$ & $\mathbf{0 , 0 0 0}$ & $12,10 \%$ \\
Dirigeant religieux & 116 & $59(50,86 \%)$ & $\mathbf{0 , 0 0 1}$ & $11,50 \%$ \\
Père & 223 & $101(45,29 \%)$ & $\mathbf{0 , 0 0 2}$ & $10,50 \%$ \\
Enseignant & 446 & $184(41,26 \%)$ & $\mathbf{0 , 0 0 5}$ & $9,70 \%$ \\
Mère & 50 & $24(48,00 \%)$ & 0,091 & $5,80 \%$ \\
Grands-parents & 219 & $88(40,18 \%)$ & 0,223 & $4,20 \%$ \\
\hline
\end{tabular}


II : Influence des autres facteurs sur le lycéen

\begin{tabular}{|c|c|c|c|c|c|}
\hline & Facteurs & $\mathrm{n}$ & $\begin{array}{c}\text { Fumeur } \\
\text { actif }\end{array}$ & $\mathrm{p}$ & $\begin{array}{c}\text { V de } \\
\text { Cramer } \\
\% \\
\end{array}$ \\
\hline $\begin{array}{c}\text { Situation } \\
\text { matrimoniale } \\
\text { des parents }\end{array}$ & $\begin{array}{c}\text { Veufs } \\
\text { Divorcés } \\
\text { Séparés } \\
\text { Mariés } \\
\text { En concubinage }\end{array}$ & $\begin{array}{c}76 \\
57 \\
146 \\
421 \\
150\end{array}$ & $\begin{array}{c}7 \\
5 \\
10 \\
26 \\
8\end{array}$ & 0,771 & $4,60 \%$ \\
\hline $\begin{array}{l}\text { Mode de vie du } \\
\text { lycéen }\end{array}$ & $\begin{array}{c}\text { Habite avec un ami } \\
\text { Habite avec un des parents } \\
\text { Habite avec les deux parents } \\
\text { Habite seul } \\
\text { Habite avec un tuteur }\end{array}$ & $\begin{array}{c}3 \\
196 \\
387 \\
32 \\
232\end{array}$ & $\begin{array}{c}1 \\
17 \\
28 \\
2 \\
8\end{array}$ & 0,065 & $10,20 \%$ \\
\hline Religion & $\begin{array}{c}\text { Traditionnelle } \\
\text { Musulmane } \\
\text { Chrétienne }\end{array}$ & $\begin{array}{c}15 \\
169 \\
666\end{array}$ & $\begin{array}{c}3 \\
15 \\
38\end{array}$ & 0,036 & $8,90 \%$ \\
\hline $\begin{array}{c}\text { Statut } \\
\text { confessionnel } \\
\text { du lycée }\end{array}$ & $\begin{array}{l}\text { Lycée privé non confessionnel } \\
\text { Lycée privé musulman } \\
\text { Lycée privé protestant } \\
\text { Lycée public } \\
\text { Lycée privé catholique }\end{array}$ & $\begin{array}{c}82 \\
79 \\
144 \\
407 \\
138\end{array}$ & $\begin{array}{c}8 \\
7 \\
12 \\
23 \\
6\end{array}$ & 0,352 & $7,20 \%$ \\
\hline Statut du lycée & $\begin{array}{l}\text { Lycées privés } \\
\text { Lycées publics }\end{array}$ & $\begin{array}{l}443 \\
407\end{array}$ & $\begin{array}{l}33 \\
23\end{array}$ & 0,291 & $3,60 \%$ \\
\hline
\end{tabular}

Tableau III : Connaissance des lycéens sur les maladies liées au tabac

\begin{tabular}{ccccccc}
\hline Maladies & $\begin{array}{c}\text { Fortement } \\
\text { d'accord\% }\end{array}$ & D'accord\% & $\begin{array}{c}\text { Incertain } \\
\%\end{array}$ & $\begin{array}{c}\text { Pas } \\
\text { d'accord\% }\end{array}$ & $\begin{array}{c}\text { Fortement } \\
\text { en } \\
\text { désaccord\% }\end{array}$ & TOTAL\% \\
\hline $\begin{array}{c}\text { Maladies } \\
\text { Cardiaques } \\
\text { Maladies }\end{array}$ & 32 & 24 & 27 & 12 & 5 & 100 \\
$\begin{array}{c}\text { vasculaires } \\
\text { Maladies }\end{array}$ & 23 & 26 & 34 & 12 & 5 & 100 \\
$\begin{array}{c}\text { respiratoires } \\
\text { Maladies } \\
\text { sexuelles }\end{array}$ & 69 & 20 & 6 & 3 & 2 & 100 \\
Cancer & 61 & 7 & 26 & 34 & 26 & 100 \\
\hline
\end{tabular}

\section{Mesures anti-tabac}

Concernant les mesures anti-tabac, 85,2\% des lycéens savaient qu’il existe des lois anti-tabac en vigueur à Madagascar ;92,9\% des lycéens savaient l'existence de règlements interdisant de fumer au sein de leur lycée ; 99,1\% savaient que des sanctions étaient prises pour ceux qui étaient attrapés 
en train de fumer dans l'enceinte du lycée. Ces sanctions faisaient peur à $69,6 \%$, ne faisaient rien à 22,3\% et faisaient rire aux 8,1\% des lycéens.

\section{Discussion}

\section{Prevalence et facteurs determinants du tabagisme}

Dans notre étude, la prévalence des lycéens fumeurs actifs était de 6,59\%. En Algérie, elle était de 8,5\% en 2015 (Elotmani, 2015). En 2008, la prévalence était de 5,7\% au Cameroun (MINSANTE du Cameroun, 2008), 3,2\% au Zimbabwe (BRTI, 2008) et 5,8\% en Sierra Léone (National School Health Services, 2008). En 2007 au Canada, la prévalence des fumeurs actifs chez les jeunes du secondaire était de $16 \%$ (Institut de la statistique du Québec, 2007). Les enquêtes dans les pays d'Amérique et d'Europe en 2006 ont estimé que 18\% des jeunes de 13 à 15 ans fumaient (CDC, 2006).

En plus, dans notre étude, l'âge moyen de la prise de la première cigarette à 13,15 ans était moins jeune que celui des Canadiens de 11,1 ans en 2009 (Payette, 2009).

Le taux de $14,05 \%$ initiés au tabac avant l'âge de 10 ans était similaire à celui de Madagascar en général en 2008 (14,6\%) (Rakotoniaina, 2008). Par contre, en Éthiopie en 2005 et en Algérie 17,5\% et 30,5\% respectivement ont été initié au tabac avant l'âge de 10 ans (DACA,2005; Ministère de la Santé de la Population et de la Réforme Hospitalière, 2007). Chez nos jeunes, le fait d'être majeur corrélait significativement avec l'utilisation du tabac. Dans l'étude malagasy de 2011, le niveau scolaire ( $3^{\mathrm{e}}$ et plus) était aussi associé à une consommation plus importante de tabac (Befinoana, 2011). Toutefois, au Sénégal en 2011, l'âge ne constituait pas un facteur déterminant l'usage du tabac chez les élèves (Faye, 2011). Les interventions en matière de lutte contre le tabagisme devraient viser les adolescents dès leur plus jeune âge.

$\mathrm{Au}$ total, les lycéens de Mahajanga fumaient globalement moins précocement que ceux des pays occidentaux et de certains pays Africains. La prévalence du tabagisme se rapprochait de celles des pays Africains, parmi celles qui étaient relativement basses. La différence avec les pays occidentaux était importante. Il est possible que dans les pays occidentaux les jeunes fumeurs soient moins sujets à la stigmatisation et qu'ils aient plus de pouvoir d'achat. Par contre il semble que la proportion des adolescents ayant déjà fumé (36,82\%) augmente. En effet même si en 2011, elle était déjà de 36,6\% dans tout Madagascar, ce chiffre était de 11,4\% dans la province de Mahajanga (Befinoana, 2011).

La prédominance masculine des fumeurs a aussi été retrouvée dans les autres études : à Madagascar en 2011 (Befinoana, 2011), au Maroc en 2014, en Algérie en 2007 ou encore au Cameroun en 2008 (Bouchra, 2014; 
Ministère de la Santé de la Population et de la Réforme Hospitalière, 2007; MINSANTE du Cameroun, 2008).

Les fumeurs qui exerçaient le plus d'influence sur le statut tabagique des lycéens étaient par ordre décroissante : ami proche, cousin, frère, oncle, dirigeant religieux, père et enseignant. Au Sénégal en 2011, tous les élèves fumeurs actifs affirmaient avoir au moins un professeur fumeur, 29\% avaient un ami proche fumeur, et $24 \%$ avaient des parents consommateurs de tabac. Chez les élèves affirmant avoir au moins un parent fumeur, il s'agissait du père dans $92 \%$, de la mère dans $2 \%$ et des deux parents dans $6 \%$ des cas (Faye, 2011). Le rôle des adultes est indéniable. En plus de leur influence, les adultes fumeurs se trouvent dans l'impossibilité d'interdire aux jeunes de fumer.

Nous avons constaté que les lycéens de religions traditionnelles malagasy fumaient significativement plus que ceux des religions musulmane et chrétienne. Ceci pourrait s’expliquer par le fait que certaines religions participent à la lutte contre le tabac plus que d'autres.

e. Pourquoi fumer ? Pourquoi avoir arrêté de fumer ?

Les motifs de la première consommation de cigarette reflètent assez bien la période de l'adolescence : essai (64,22\%), mode et quelquefois révolte (en réponse à une interdiction).

La majorité des ex-fumeurs (74,71\%) ont arrêté de fumer par peur des maladies liées au tabac. Notre résultat était similaire à celui de l'étude réalisée en France en 2003 qui retrouvait que 74,25\% des lycéens étaient plus sensibles à la peur de la mort (Michaud, 2003).

La relation entre tabagisme et maladies respiratoires et les cancers est assez bien acceptée par nos élèves, comme dans les autres pays. Par contre, son rôle dans la survenue des maladies cardio-vasculaires est beaucoup moins connu. Au Sénégal en 2011, les élèves connaissaient au moins un méfait du tabac dans 86\% des cas. Le cancer, les pneumopathies, les cardiopathies étaient parmi les méfaits du tabac les plus connus et cités respectivement par 42\%, 40\%, 21\% des élèves (Faye, 2011). En France en 2003, le cancer était évoqué par 96\% des lycéens comme étant la maladie liée à l'usage du tabac (Michaud, 2003). Il est important d'informer les jeunes sur le fait que les effets nocifs du tabac ne se limitent pas aux organes directement exposés à la fumée.

\section{Dépendance}

En ce qui concerne la dépendance, $75 \%$ des fumeurs étaient peu ou pas dépendants au tabac contre 81\% au Sénégal en 2011 (Faye, 2011). Le reste était moyennement à fortement dépendant. La prédominance des non ou peu dépendants s'explique probablement par le fait que la plupart des lycéens ont fumé depuis peu de temps. 


\section{Achat de tabac}

Les lycéens pouvaient se procurer facilement de cigarettes. Cela est dû à leur prix abordable et au fait que le vendeur ne demande pas l'âge de l'acheteur. Une proportion importante de lycéens (60\%) ont trouvé que la cigarette était soit abordable (35\%) soit bon marché (25\%). Pourtant les adolescents sont sensibles aux prix des cigarettes (Befinoana, 2011).

\section{Conclusion}

La prévalence du tabagisme actif des lycéens de 6,59\% était plus faible que celle de la plupart des études réalisées que ce soit à Madagascar, en Afrique ou dans les pays occidentaux. Par contre 36,82\% avaient déjà fumé au moins une fois dans leur vie. Ce qui est plutôt inquiétant, d'autant plus que la dépendance au tabac peut s’installer rapidement chez l'adolescent. Certains facteurs ont été corrélés positivement avec le fait d’être fumeur. Le comportement des lycéens reflète les difficultés qu'ils rencontrent durant l'adolescence (influences de l'entourage, indiscipline, essai et aventure). Le rôle des adultes ne consistera pas seulement à appliquer les lois en vigueur et les disciplines, ou augmenter le prix des cigarettes mais surtout à donner un bon exemple en matière de lutte contre le tabagisme.

\section{References :}

Befinoana, Razanamihaja, N. (2011). Tabagisme et facteurs associés chez les adolescents scolarisés à Madagascar. Santé Publique, 6(23), 465-474.

Bejot, Y., Caillier, M., Rouaud, O., Benatru, I., Maugras, C., Osseby, G. V. \& Giroud, M. (2007). Épidémiologie des accidents vasculaires cérébraux : Impacts sur la décision thérapeutique. Presse Médicale, 36 (1, Part 2), 117-27.

Bouchra, L., Latifa, B., Majda, L., Noureddine, E. (2014). Tabagisme chez les collégiens de la region de fes-boulemane (Maroc). European Scientific Journal, 2014, 10 (9), 50-61.

BRTI (Biomedical Research \& Training Institute) Harare. (2008). Report of the global youth tobacco survey (GTYS) Zimbabwe-1999, 2003 \& 2008 Adolescents aged 13-15 years. Retrieved from http://www.afro.who.int/index.php?option=com_docman\&task=doc_downlo ad\&gid $=1903$.

CDC (Centers for Disease Control and Prevention). (2006). Use of cigarettes and other tobacco products among students aged 13-15 years--worldwide, 1999-2005. Morbidity and Mortality Weekly Report, 2006, 55 (20), 553-6.

CREAM (Centre de Recherches, d'Études et d'appui à l'Analyse Economique à Madagascar). Monographie Région Boeny. (2014). Retrieved from http://www.monographiemada.com/docpdf/Boeny/BOENY.pdf 
DACA (Drug Administration and Control Authority) of Ethiopia. (2005). A report on Global Youth Tobacco Survey (GYTS) Conducted in Secondary Schools in Addis Ababa-Ethiopia. Retrieved from http://www.afro.who.int/index.php?option=com_docman\&task=doc_downlo ad\&gid $=5963$.

Elotmani, F. Z., Elbiaze, M., Amara, B., Serraj, M., Benjelloun, M. C. (2015). Comportements, attitudes, et connaissances des écoliers vis-à-vis du tabagisme à la ville de Fès, Revue des Maladies Respiratoires, 32, 137.

Faye, A., Seck, I., Seye Ndiaye, A. C., Ndiaye, P., Tal-dia, A. (2011). Aspects épidémiologiques du tabagisme en milieu scolaire dakarois, au Sénégal. Médecine d’Afrique Noire, 58 (7), 363-9.

INPES, Ministère chargé de la Santé (France). (2015). Évaluation de la dépendance chimique à la nicotine : questionnaire de Fagerström. Retrieved from: https://www.tabac-infoservice.fr/var/storage/upload/Q1_Fagerstrom.pdf

Institut de la statistique du Québec. (2007). Aperçu des habitudes tabagiques et de l'exposition à la fumée de tabac dans l'environnement des enfants, des jeunes et des adultes québécois. Zoom santé. 2007; 1-8. Retrieved from www.stat.gouv.qc.ca

Michaud, C., Saraiva, I., Henry, Y., Dodane, M. (2003). Tabac : connaissances, motivations et souhaits de lycéens du Doubs. Réflexions pour la prévention. Santé Publique, 15 (1), 69-78.

Ministère de la Santé de la Population et de la Réforme Hospitalière. (2007). Enquête globale sur le tabagisme chez les jeunes (G.Y.T.S) Algérie. Retrieved

from http://www.who.int/fctc/reporting/party_reports/algeria_annex2_gyts_2007_ fr.pdf.

MINSANTE du Cameroun. (2008). Enquête globale sur le tabagisme en milieu jeune (GYTS 2008). Retrieved from http://www.afro.who.int/index.php?option=com_docman\&task=doc_downlo ad\&gid $=1912$.

National School Health Services, Ministry of Health and Sanitation. (2008). The Sierra Leone Global Youth Tobacco Survey Report (GYTS). Retrieved from

http://apps.who.int/fctc/implementation/database/sites/implementation/files/d ocuments/reports/sierra_leone_annex2_gyts_2008.pdf.

Payette, Y., Nguyen, C. Institut national de santé publique du Québec. (2009). Enquête sur le tabagisme chez les jeunes 2004-2005 Comparaisons Québec-Canada. Retrieved from http://www.inspq.qc.ca

Rakotoniaina, J. P. (2008). Enquête nationale sur le tabagisme chez les jeunes

(GYTS).

Retrieved

from 
http://www.who.int/fctc/reporting/party_reports/madagascar_annex2_GYTS 2008.pdf.

WHO (World Health Organization). (2014). Tobacco. Fact sheet $N^{\circ} 339$. Retrieved from http://www.who.int/mediacentre/factsheets/fs339/en/ 